\title{
Production of high cellulase yields from polypore fungi in solid-state fermentation using green tea waste Nguyen $\mathrm{KA}^{1,2}$, Penkhrue $\mathrm{W}^{3}$ and Lumyong $S^{1,3,4^{*}}$
}

This manuscript has been withdrawn by the authors as the previous text was prematurely uploaded and requires some additional experiments that may alter the conclusions. Therefore, the manuscript should not be cited in reference for this project. Please contact the corresponding author if you have any questions. 